\title{
A 21-year-old man with systemic-onset juvenile rheumatoid arthritis, cough and progressive dyspnea
}

\author{
A Leber MD ${ }^{1}$, S Carette MD MPhil FRCPC ${ }^{2}$, KR Chapman MSc MD FRCPC FCCP3, DM Hwang MD PhD FRCPC ${ }^{4}$, \\ LG Singer MD FRCPC FCCP5, TK Marras MD FRCPC FCCP6
}

\begin{abstract}
A Leber, S Carette, KR Chapman, DM Hwang, LG Singer, TK Marras. A 21-year-old man with systemic-onset juvenile rheumatoid arthritis, cough and progressive dyspnea. Can Respir J 2010;17(3):e42-e44.

Primary or nonobstructive, endogenous lipoid pneumonia is a rare clinical entity usually associated with an underlying systemic disease. The present report describes a case involving a 21-year-old man with systemic-onset juvenile rheumatoid arthritis who developed primary endogenous lipoid pneumonia. Multiple treatment regimens were attempted; however, definitive management was only achieved through double-lung transplantation.
\end{abstract}

Key Words: Endogenous lipoid pneumonia; Rheumatoid arthritis

\section{Un homme de 21 ans ayant une arthrite rhumatoïde juvénile à apparition systémique, une toux et une dyspnée évolutive}

\begin{abstract}
La pneumonie lipoïde endogène primaire non obstructive est une entité clinique rare généralement associée à une maladie systémique sous-jacente. Le présent rapport décrit le cas d'un homme de 21 ans ayant une arthrite rhumatoïde juvénile à apparition systémique qui a souffert d'une pneumonie lipoïde endogène primaire. On a tenté de multiples schémas posologiques, mais on est parvenu à une prise en charge définitive seulement après une double greffe pulmonaire.
\end{abstract}

\section{CASE PRESENTATION}

A 21-year-old Canadian-born man was referred for a two-year history of nonproductive, nocturnal cough and slowly progressive exertional dyspnea. His medical history was significant for systemic-onset juvenile rheumatoid arthritis (SoJRA), which was diagnosed at four years of age. SoJRA is a subset of JRA and is characterized by intermittent fever, rash and joint involvement. The respiratory symptoms coincided with an acute flare of his arthritis, which had been quiescent for two years. He suffered from several steroid-related complications including gastroesophageal reflux disease, osteoporosis, growth failure and cataracts. His medications consisted of prednisone, infliximab, indomethacin, alendronate and omeprazole. He had a five-year history of methotrexate use, which was discontinued eight months before the initial assessment.

On physical examination, his vital signs were normal and he had a Cushingoid appearance. Respiratory auscultation revealed bilateral, basilar inspiratory crackles. His cardiac and abdominal examinations were unremarkable. He had bilateral flexion contractures in his elbows, hips and knees. Both elbows were swollen from active joint disease.

His complete blood count demonstrated normocytic anemia, with a hemoglobin level of $104 \times 10^{9}$ cells/L, leukocytosis (white blood cell count $24.5 \times 10^{9}$ cells/L) and thrombocytosis (platelet count of $489 \times 10^{9}$ cells/L). Other routine blood tests were unremarkable. The initial chest $\mathrm{x}$-ray revealed prominent interstitial markings throughout the lungs, with some airspace opacification. A restrictive pattern was seen on pulmonary function testing (total lung capacity of $68 \%$ of predicted, forced vital capacity of $53 \%$ of predicted, forced expiratory volume in $1 \mathrm{~s} /$ forced vital capacity ratio of 1.4/1.62 [98\% of predicted]) with a decreased diffusion capacity of $35 \%$ of predicted. An initial computed tomography scan showed interlobular thickening and ground-glass opacities, predominantly in the lung bases (Figure 1A). Bronchoscopy was negative for bacterial, viral and fungal pathogens. A bronchoalveolar lavage cellular assessment was limited by mixed airway and alveolar sampling, but was suggestive of acute inflammation.

The patient's symptoms were persistent four months later, and a subsequent computed tomography scan revealed obvious progression of the previously observed abnormalities (Figure 1B). The differential diagnosis at that time encompassed infection, including opportunistic organisms (due to his use of immunosuppressant medications), drug reactions, particularly methotrexate-induced pneumonitis and JRA-related interstitial lung disease. To obtain a definitive diagnosis, a video-assisted thoracoscopic biopsy was performed. The pathological examination revealed filling of the small airways and surrounding airspaces with eosinophilic material containing numerous cholesterol clefts and foamy macrophages (Figure 2). These findings were associated with moderate inflammatory infiltrates composed of lymphocytes, plasma cells and histocytes. Focal areas displayed intra-alveolar eosinophilic granular material that was somewhat reminiscent of lipoproteinosis. Stains for mycobacteria and fungi were negative, as were all lung tissue cultures. A videofluoroscopic swallow study did not demonstrate aspiration as a possible etiology for this disorder. Hence, a diagnosis of endogenous lipoid pneumonia (of the primary or nonobstructive type) was made.

${ }^{1}$ Department of Medicine, University Health Network; ${ }^{2}$ Division of Rheumatology, Department of Medicine; ${ }^{3}$ Division of Respirology, Department of Medicine, University Health Network and Mount Sinai Hospital; ${ }^{4}$ Department of Pathology, University Health Network; ${ }^{5}$ Division of Respirology, Department of Medicine, University Health Network and University of Toronto; ${ }^{6}$ Division of Respirology, Department of Medicine, University Health Network and Mount Sinai Hospital, University of Toronto, Toronto, Ontario

Correspondence: Dr Theodore K Marras, Division of Respirology, University Health Network and Mount Sinai Hospital, Toronto Western Hospital, 399 Bathurst Street, 7E-452, Toronto, Ontario M5T 2S8. Telephone 416-603-5767, fax 416-603-5375,

e-mail ted.marras@uhn.on.ca or ted.marras@utoronto.ca 

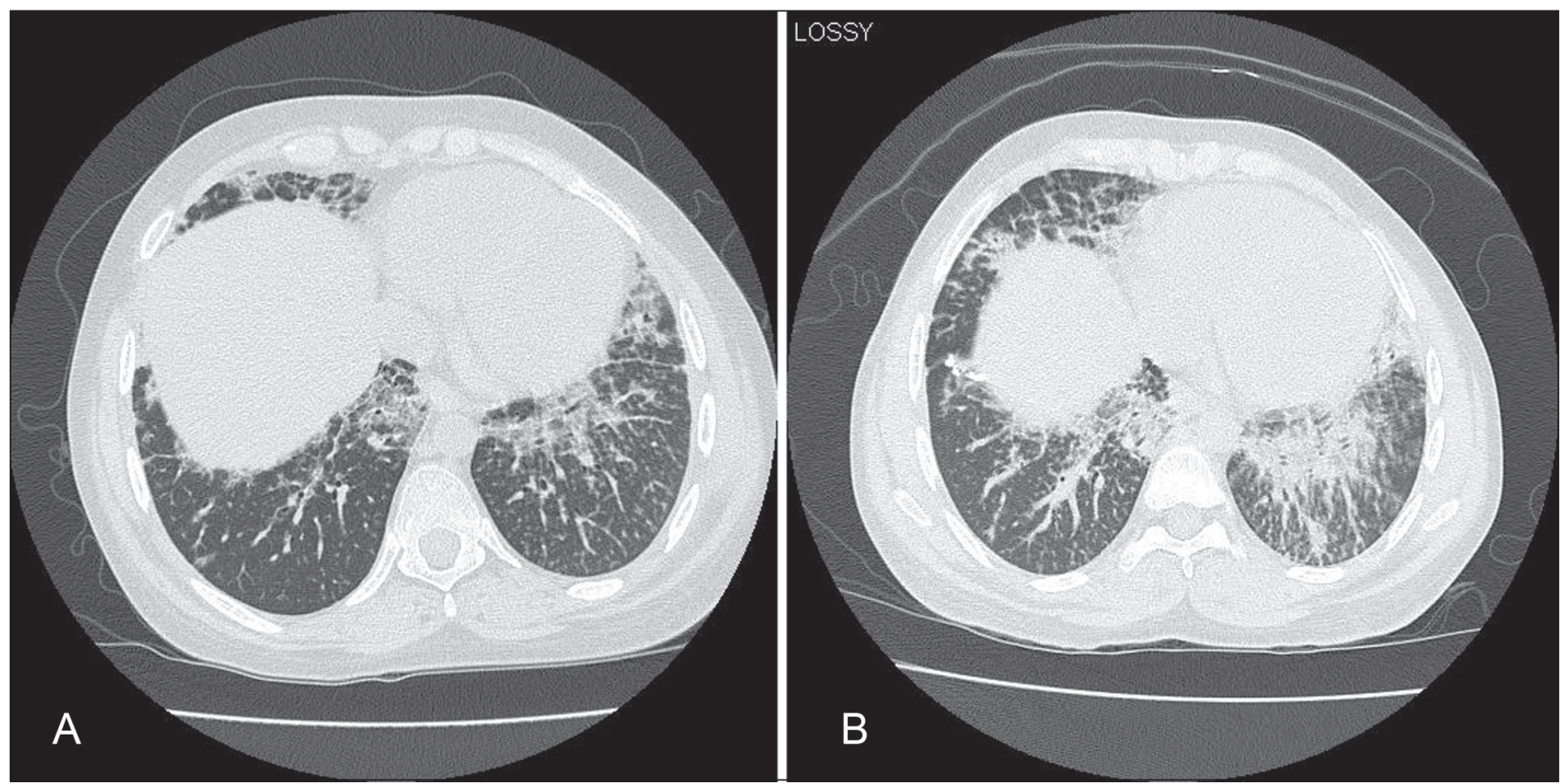

Figure 1) Computed tomogram of the chest at presentation (A) and four months later (B), demonstrating obvious progression of ground-glass opacities and extensive interlobular thickening in a 'crazy paving' pattern

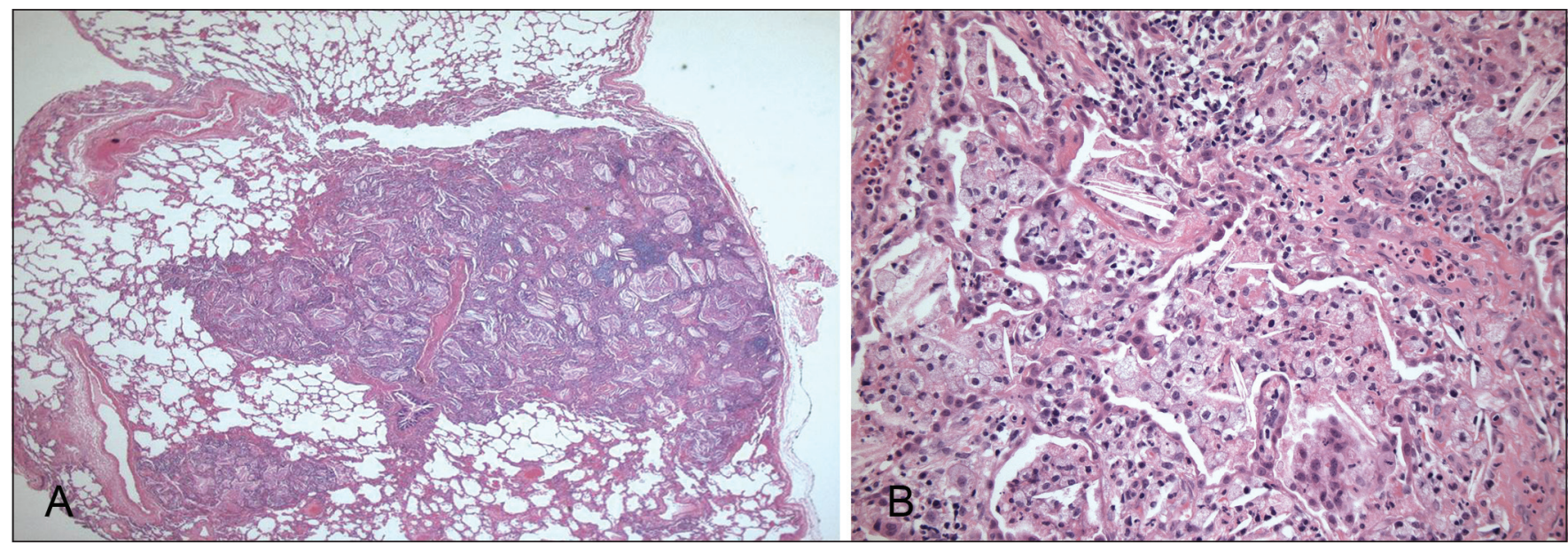

Figure 2) Lung biopsy. A Low-power magnification showing nodular areas with air space filling (hematoxylin and eosin stain, original magnification $\times 16$ ). B High-power magnification showing intra-alveolar cholesterol crystals and foamy macrophages (hematoxylin and eosin stain, original magnification $\times 200$ )

\section{DISCUSSION}

Lipoid pneumonia consists of airspace filling with lipid material and an associated inflammatory reaction. It is generally classified as exogenous or endogenous. Exogenous lipoid pneumonia is associated with aspiration of lipid material, including mineral oil laxatives, kerosene and lipids inhaled during metal processing, stimulating a foreign body-type reaction to lipid material in the lung parenchyma. Endogenous lipoid pneumonia is caused by the release of lipid material from alveolar cell walls, with a subsequent inflammatory response believed to be related to increased lipid uptake by alveolar macrophages (1). The most common cause is bronchial obstruction (secondary to a malignancy or foreign body) resulting in necrosis of distal alveolar cells and subsequent release of lipid material (1). It has also been described in case reports in association with undifferentiated connective tissue disease (2), hypercholesterolemia secondary to primary sclerosing cholangitis (1) and in one patient with SoJRA (3). In the absence of bronchial obstruction, the disorder may be called primary (or nonobstructive) endogenous lipoid pneumonia. Drug reactions must always be considered in patients with undiagnosed lung problems, especially in the context of complex drug therapies. Regarding the current case, because we were unaware of any reports of endogenous lipoid pneumonia caused by drugs, and because our patient did not improve despite multiple medication changes, we do not believe that a drug reaction caused his lung disease.

The pathogenesis of endogenous lipoid pneumonia in the absence of bronchial obstruction is unclear. It has been hypothesized that it shares a pathogenesis similar to that of pulmonary 
alveolar proteinosis (PAP). Indeed, the two entities have been found to coexist in the same patient (4). In PAP, there is an accumulation of lipoproteinaceous material in the airways due to alveolar macrophage dysfunction leading to ineffective clearance. Pulmonary inflammation and fibrosis, seen in lipoid pneumonia, is not prominent in PAP but has been documented (5). Alveolar macrophage abnormalities have also been documented in rheumatoid arthritis, particularly with increased cellular activation and inflammatory cytokine production (2). Sato et al (4) hypothesized that in endogenous lipoid pneumonia, an unknown stimulus inhibits the clearance of alveolar lipids by alveolar macrophages. In SoJRA-related endogenous lipoid pneumonia, alveolar macrophage upregulation possibly results in a detrimental inflammatory response to the normal release of lipids in the airway.

Clinically, endogenous lipoid pneumonia presents most commonly with a subacute onset of nonproductive cough and progressive dyspnea. The radiological appearance is heterogeneous, with the most common patterns being low attenuation consolidation, ground-glass opacities and so-called 'crazy paving'. Cytological findings from bronchoscopic lavage demonstrate a predominance of foamy macrophages containing large fatty vesicles (1). Pathological findings include interstitial and intra-alveolar cholesterol granulomas (3), lipid-laden macrophages (4) and interstitial inflammation (1). Although our patient's biopsy contained a relative abundance of cholesterol clefts, the otherwise characteristic findings strongly support the diagnosis of endogenous lipoid pneumonia. It is interesting to note that the label of progressive pulmonary interstitial and intra-alveolar cholesterol granulomas has been used to describe cases similar to ours $(3,4)$. In both of these earlier cases, cholesterol clefts were indeed prominent. We prefer the term endogenous lipoid pneumonia for its simplicity, recognizing that the primary (nonobstructive) type may well display more prominent cholesterol clefts. Focal areas also displayed intraalveolar eosinophilic granular material that was somewhat

\section{REFERENCES}

1. Berghaus T, Haeckel T, Wagner T, von Scheidt W, Schwaiblmair M. Endogenous lipoid pneumonia associated with primary sclerosing cholangitis. Lancet 2007;369:1140.

2. Barta Z, Szabo GG, Bruckner G, Szegedi G. Endogenous lipoid pneumonia associated with undifferentiated connective tissue disease. Med Sci Mon 2001;7:134-6.

3. Schultz R, Mattila J, Gappa M. Development of progressive pulmonary interstitial and intra-alveolar cholesterol granulomas reminiscent of lipoproteinosis, but the diagnosis of endogenous lipoid pneumonia was overwhelmingly favoured.

Treatment options are limited and the best choice remains unclear. Case reports have described successful treatment with total lung lavage (1), systemic steroids (2) or tumour necrosis factor-alpha antagonists (3). Multiple treatment courses were attempted in our patient, including high-dose pulsed steroids, infliximab and long-term azithromycin. Granulocyte macrophage-colony stimulating factor (GM-CSF), although used in PAP, was not considered due to the minimal histological coexistence of PAP on biopsy, the lack of data on the use of GM-CSF in endogenous lipoid pneumonia and the risk of macrophage activation syndrome with the use of GM-CSF in patients with SoJRA. Whole lung lavage was considered but not pursued because of the scarcity of evidence supporting its use, and the substantial risk associated with both the lavage itself and securing the patient's airway due to cervical instability and retrognathia. His pulmonary disease was further complicated by Staphylococcus aureus pneumonia and abscess. He began to require supplemental oxygen. Despite treatment, his lung function continued to deteriorate, eventually requiring a double-lung transplant. His grafted lungs remained radiologically clear and lung function was normal one year later. The final pathological examination of the explanted lungs was consistent with endogenous lipoid pneumonia.

To our knowledge, this is the first reported case of lung transplantation for the treatment of endogenous lipoid pneumonia. It is unclear whether this intervention will prove to be definitive management. There is one documented case in the literature (5) of PAP recurrence three years after a double-lung transplant that was attributed to ongoing alveolar macrophage dysfunction. If our patient's endogenous lipoid pneumonia was due to alveolar macrophage dysfunction associated with SoJRA, then it is imperative to remain vigilant for possible disease recurrence.

(PICG) associated with therapy-resistant chronic systemic juvenile arthritis. Ped Pulm 2001;32:397-402.

4. Sato K, Takahashi H, Amano H, Uekusa T, Dambara T, Kira S. Diffuse progressive pulmonary interstitial and intra-alveolar cholesterol granuloma in childhood. Eur Respir J 1996;9:2419-22.

5. Parker L, Novotny D. Recurrent alveolar proteinosis following double lung transplantation. Chest 1997;111:1457-8. 


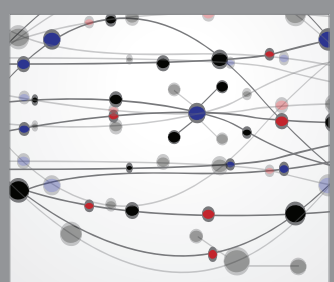

The Scientific World Journal
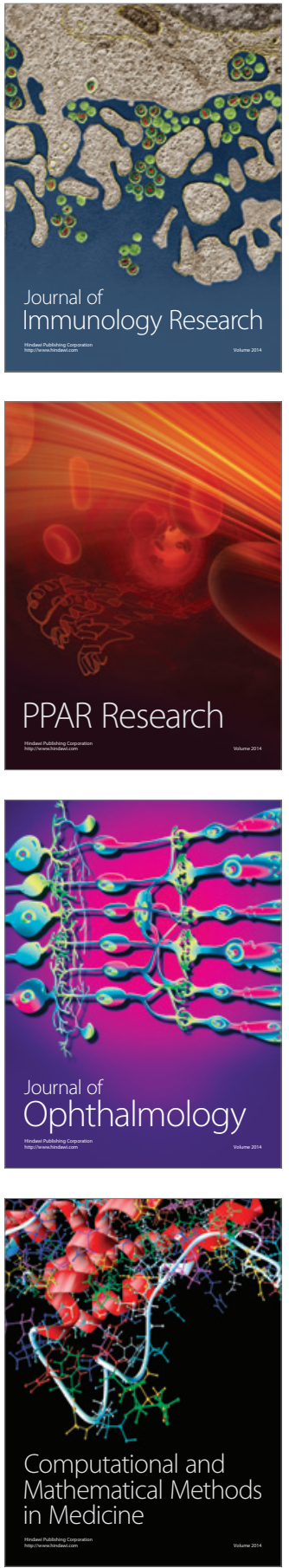

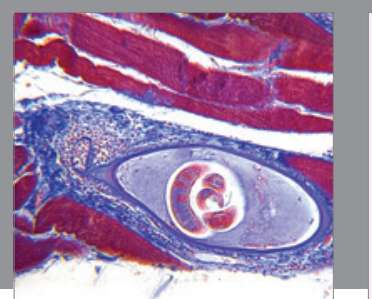

Gastroenterology Research and Practice

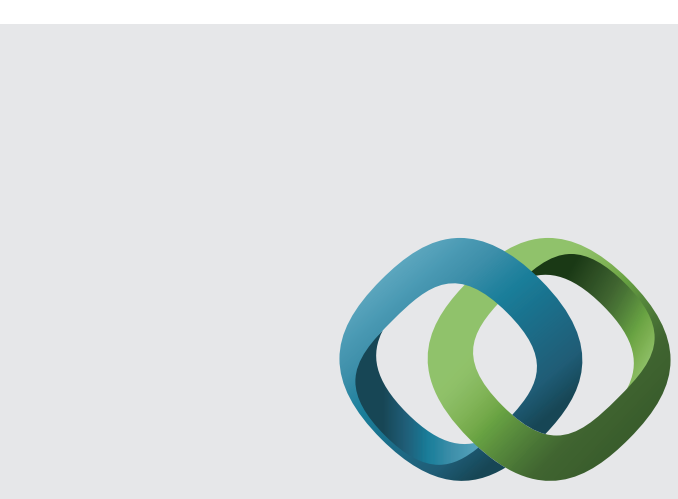

\section{Hindawi}

Submit your manuscripts at

http://www.hindawi.com
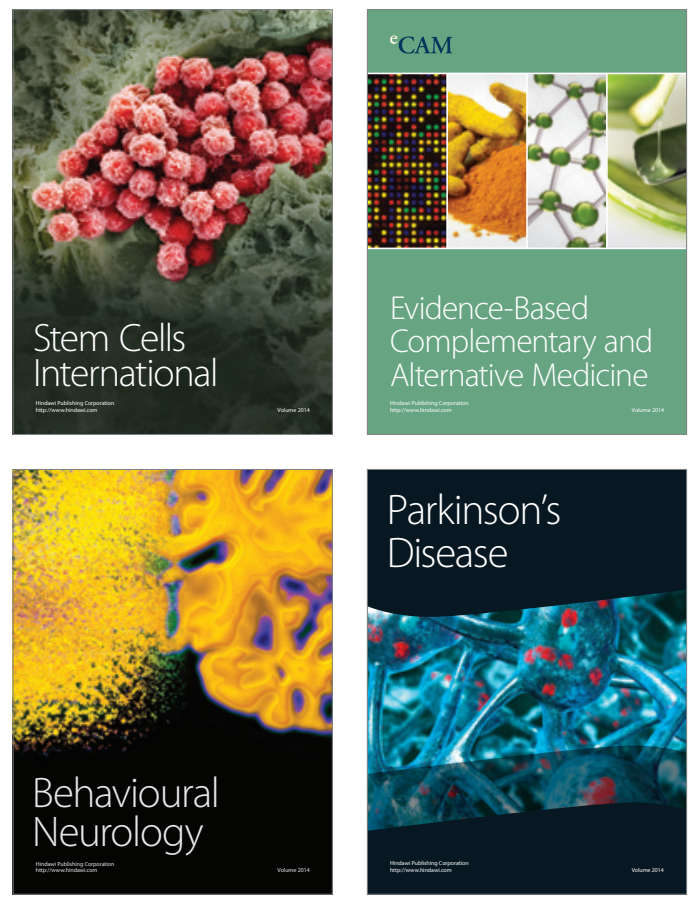
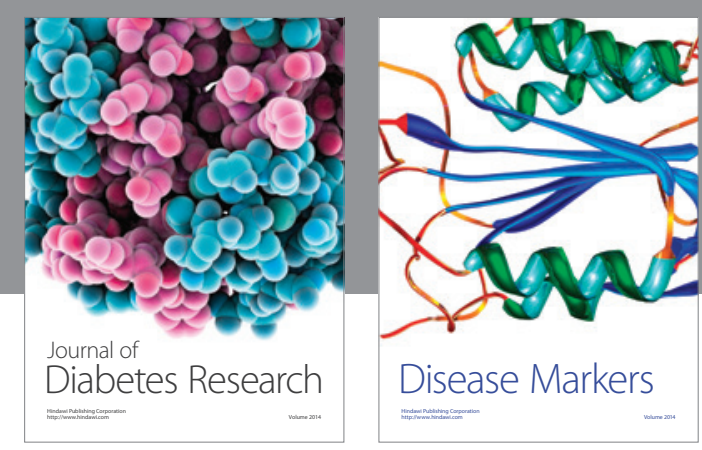

Disease Markers
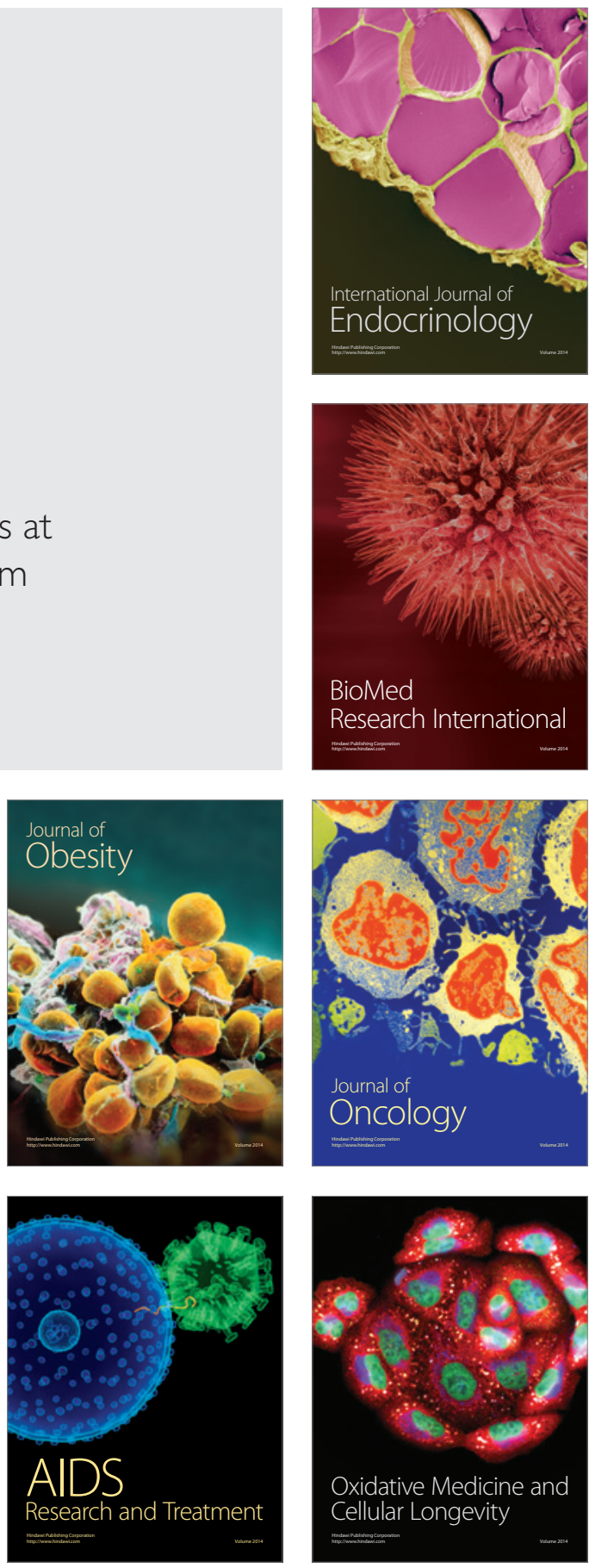